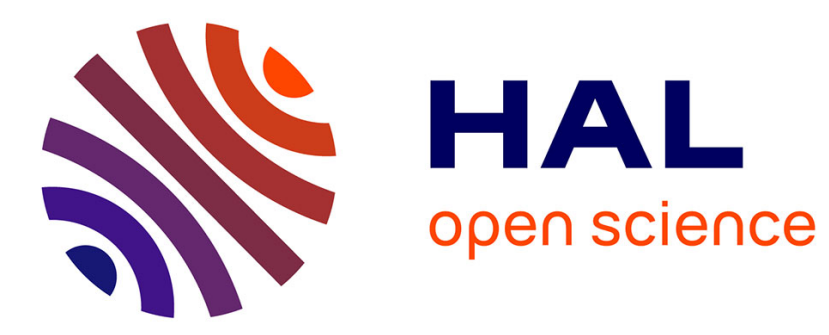

\title{
Transfer matrix calculation of the exponent $\gamma$ for two-dimensional self-avoiding walks
}

\author{
H. Saleur, Bernard Derrida
}

\section{To cite this version:}

H. Saleur, Bernard Derrida. Transfer matrix calculation of the exponent $\gamma$ for two-dimensional selfavoiding walks. Journal of Statistical Physics, 1986, 44 (1-2), pp.225-235. 10.1007/BF01010914 . hal-03285911

\section{HAL Id: hal-03285911 \\ https://hal.science/hal-03285911}

Submitted on 21 Jul 2021

HAL is a multi-disciplinary open access archive for the deposit and dissemination of scientific research documents, whether they are published or not. The documents may come from teaching and research institutions in France or abroad, or from public or private research centers.
L'archive ouverte pluridisciplinaire HAL, est destinée au dépôt et à la diffusion de documents scientifiques de niveau recherche, publiés ou non, émanant des établissements d'enseignement et de recherche français ou étrangers, des laboratoires publics ou privés. 


\title{
Transfer Matrix Calculation of the Exponent $\gamma$ for Two-Dimensional Self-Avoiding Walks
}

\author{
H. Saleur ${ }^{1}$ and B. Derrida ${ }^{1}$
}

Received December 13, 1985

We develop two independent transfer matrix methods for the determination of the exponent $\gamma$ in the two-dimensional, self-avoiding walk problem. Our first method is based on the calculation of the correlation length and uses conformal invariance. Our second method is based on the direct calculation of the moments of the order parameter distribution. Our results are in good agreement with the conjectured values.

KEY WORDS: Polymers; self-avoiding walks; transfer matrix.

The problem of self-avoiding walks (SAW) on a lattice is one of the most studied problems in statistical mechanics. The basic quantities to be determined are the exponent $v$, which relates the mean square end-to-end distance $\left\langle\left|\mathbf{R}_{l}\right|^{2}\right\rangle$ to the length $l$ of the walk for $l \gg 1$ by

$$
\left\langle\left|\mathbf{R}_{l}\right|^{2}\right\rangle \sim l^{2 v}
$$

the connectivity constant $\mu$ and the exponent $\gamma$, which give the asymptotic behavior of the number of SAWs of length $l$ and fixed origin

$$
\omega_{I} \sim \mu^{l} l^{\gamma-1}
$$

The critical exponents $\gamma$ and $v$ are expected to be universal (they should depend only on the dimensionality of the lattice) as for magnetic systems $^{(1,2)}$ while the connectivity constant $\mu$ plays the role of a critical temperature in magnetic systems and thus depends on the lattice.

In two dimensions much information concerning these quantities has been obtained by numerical methods like series expansions, ${ }^{(3-5)}$ Monte

\footnotetext{
${ }^{1}$ Service de Physique Théorique, CEA-SACLAY, 91191 Gif-sur-Yvette Cedex, France.
} 
Carlo calculations, ${ }^{(6,7)}$ or real space renormalizations. ${ }^{(8-10)}$ Exact values of the exponents have also been conjectured. ${ }^{(11)}$ The value $v=\frac{3}{4}$, which was also proposed by Flory ${ }^{(12)}$ many years ago, is now considered to be in good agreement with numerical data (see Ref. 7 for a complete discussion). The value $\gamma=43 / 32^{(11)}$ has led to some discussion because on the basis of series methods, $\gamma$ was believed to be $\frac{4}{3}$ for many years. ${ }^{(3)}$ However, a reanalysis of the series expansions ${ }^{(4,5)}$ now supports the value $\gamma=43 / 32$, which agrees also with recent Monte Carlo simulations. ${ }^{(7)}$ The real space renormalization approaches were first used to determine $\mu$ and $v$ only. ${ }^{(8-10)}$ However, a cell method has been recently extended ${ }^{(10)}$ to the determination of $\gamma$, but with rather inaccurate results. The purpose of the present work is to extend the transfer matrix approach of Ref. 8 to the calculation of the exponent $\gamma$ in the SAW problem. We use two different methods. The first is based on the calculation of the correlation length and uses conformal invariance; the second is based on the direct calculation of the moments of the order parameter distribution.

In transfer matrix studies of critical phenomena one usually calculates the correlation length $\xi_{n}$ for a strip of width $n$ as a function of the inverse temperature $\beta$. One then makes a finite size scaling assumption ${ }^{(13)}$

$$
\xi_{n} \sim n f\left[n^{1 / v}\left(\beta-\beta^{c}\right)\right]
$$

which should be valid for $n \gg 1$ and $\beta-\beta^{c} \ll 1 . v$ is the correlation length exponent and $\beta^{c}$ is the inverse of the critical temperature $\beta^{c}=1 / \tau^{c}$. Estimates $\beta_{n}^{c}$ of $\beta^{c}$ can be obtained by solving the phenomenological renormalization equations ${ }^{(13)}$

$$
\frac{\xi_{n}\left(\beta_{n}^{c}\right)}{n}=\frac{\xi_{n-1}\left(\beta_{n}^{c}\right)}{n-1}
$$

while estimates of $v$ are given by

$$
1+\frac{1}{v_{n}}=\frac{\log \left[\frac{d \xi_{n}}{d \beta}\left(\beta_{n}^{c}\right) / \frac{d \xi_{n-1}}{d \beta}\left(\beta_{n}^{c}\right)\right]}{\log [n / n-1]}
$$

According to the finite size scaling form (3), these estimates 'should converge to the correct values when $n \rightarrow \infty$. In practice, one calculates numerically $\beta_{n}^{c}$ and $v_{n}$ for increasing sizes $n$ and one extrapolates these values. This method has already been applied to the determination of $\beta^{c}=1 / \mu$ and $v$ in the case of the SAW problem in Ref. 8 . 
The knowledge of the correlation length on strips also allows us to determine another exponent. From conformal invariance in two dimensions one knows that ${ }^{(14)}$

$$
\lim _{n \rightarrow \infty} \frac{\xi_{n}\left(\beta^{c}\right)}{n}=\frac{1}{\pi \eta}
$$

where $\xi_{n}$ is the correlation length of a strip of width $n$ with periodic boundary conditions and the exponent $\eta$ is related to $\gamma$ and $v$ by $\gamma / v=2-\eta$.

We use relation (5) to obtain a first determination of the ratio $\gamma / v$. We have just reproduced the calculation described in Ref. 8 to obtain estimates $\eta_{n}$ of $\eta$ by

$$
\eta_{n}=\frac{n}{\xi_{n}\left(\beta_{n}^{c}\right) \pi}
$$

The values of $\beta^{c}$ and $v$ obtained in this way have been published. ${ }^{(8)}$ These estimates are given in Table I.

All our calculations have been done for the square lattice and for strips oriented parallel to one axis of the lattice $((1,0)$ direction $)$ or for strips oriented parallel to the $(1,1)$ direction. Such diagonal strips have already been used in transfer matrix calculations. ${ }^{(15)}$

The results in Table I converge in a regular way to the expected value $^{(11)} \eta=5 / 24=0.20833$, the convergence being much better in the case of diagonal strips. To extrapolate these data we use the a posteriori extrapolation method described in Ref. 15.

Table I. Estimate of $\eta_{n}$ and $\tilde{\eta}_{n}{ }^{a}$

\begin{tabular}{rcccc}
\hline$n$ & $\begin{array}{c}\eta, \text { Normal } \\
\text { direction }\end{array}$ & $\begin{array}{c}\eta \text {, Diagonal } \\
\text { direction }\end{array}$ & $\begin{array}{c}\tilde{\eta} \text {, Normal } \\
\text { direction }\end{array}$ & $\begin{array}{c}\tilde{\eta} \text {, Diagonal } \\
\text { direction }\end{array}$ \\
\hline 2 & 0.336167 & 0.159626 & & \\
3 & 0.291922 & 0.193194 & 0.674666 & 0.206151 \\
4 & 0.257065 & 0.200920 & 0.163800 & 0.208024 \\
5 & 0.236704 & 0.204010 & 0.202772 & 0.208257 \\
6 & 0.225882 & 0.205540 & 0.208644 & 0.208309 \\
7 & 0.220063 & 0.206400 & 0.209600 & \\
8 & 0.216731 & & 0.209453 & \\
9 & 0.214673 & & 0.209146 & \\
10 & 0.213110 & & & \\
Expected value & & & 5 & \\
\end{tabular}

${ }^{a}$ In this table we give estimates $\eta_{n}$ defined by (6) in the case of the square lattice and strips oriented in normal or diagonal direction and estimates $\tilde{\eta}_{n}$ deduced from the preceeding values and an a posteriori analysis (see text). 
To do so we calculate for each triplet of results $\eta_{n-1}, \eta_{n}, \eta_{n+1}$ an "effective" exponent $\omega_{n}$ of correction to scaling by

$$
\frac{\eta_{n-1}-\eta_{n}}{\eta_{n}-\eta_{n+1}}=\frac{(n-1)^{-\omega_{n}}-n^{-\omega_{n}}}{n^{-\omega_{n}}-(n+1)^{-\omega_{n}}}
$$

Once $\omega_{n}$ is determined one extrapolates the straight line which goes through $\eta_{n-1}, \eta_{n}, \eta_{n+1}$ in the plot of $\eta_{n}$ versus $n^{-\omega_{n}}$ to obtain a new value, $\tilde{\eta}_{n}$ which is usually a better estimate of $\eta$ than $\eta_{n}$.

Our results for $\tilde{\eta}_{n}$ are also given in Table I. The convergence is now faster, particularly in the case of diagonal strips. The use of an a priori method $^{(15)}$ gave very similar results here. For the final extrapolation we have plotted in Fig. 1 all our results for $\eta_{n}$ and $\tilde{\eta}_{n}$ versus $n^{-2,5}$, a choice which gives rather smooth curves for all the data. From Fig. 1 one deduces

and thus

$$
\eta=0.2082 \pm 0.0008
$$

$$
\frac{\gamma}{v}=1.7918 \pm 0.0008
$$

This gives a relative error of about $510^{-4}$ for this ratio.

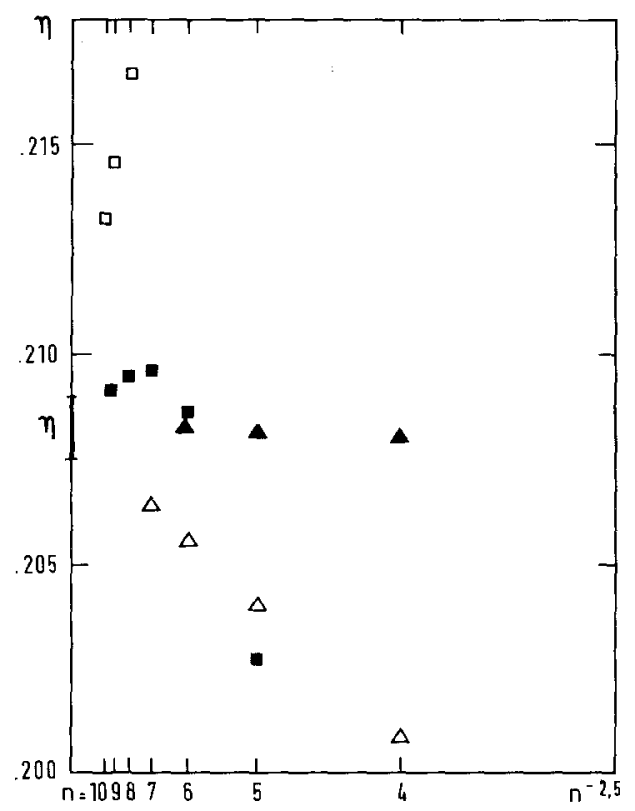

Fig. 1. Plot of different estimates of $\eta$ versus $n^{-2,5}$ (see Table I): $\square$-strips in normal direction; $\triangle$-strips in diagonal direction; - -a posteriori results, strips in normal direction; A-a posteriori results, strips in diagonal direction. The result of our final extrapolation is indicated with error bars. 
Our second approach consists of calculating the cumulants of the order parameter distribution instead of the correlation lengths. This approach was first used in Monte Carlo simulations ${ }^{(16)}$ but it is also possible to calculate the moments of the order parameter using transfer matrices. This was done for the Ising Model, ${ }^{(17,18)}$ and the estimates of the critical point and of the exponents were comparable in accuracy to those obtained with correlation lengths. Moreover the method allowed the direct determination of two independent critical exponents. We present here a similar calculation in the case of the SAW problem.

A simple way of defining the moments of the order parameter is to consider that the SAW problem is the limit of the Heisenberg model with $s$ components in the limit $s \rightarrow 0$. For a system of $s$ components classical spins whose Hamiltonian is

$$
\beta H=-\beta \sum_{\langle i j\rangle} \mathbf{S}_{i} \cdot \mathbf{S}_{j}-h \sum_{i} S_{i}^{1}
$$

with $\sum_{x=1}^{s}\left(S_{i}^{\alpha}\right)^{2}=s$ on a strip of width $n$ we define ${ }^{(17)}$ the first cumulants per unit length

$$
\begin{aligned}
& m_{2}=\lim _{L \rightarrow \infty} \frac{\left\langle M^{2}\right\rangle}{L} \\
& m_{4}=\lim _{L \rightarrow \infty} \frac{\left\langle M^{4}\right\rangle-3\left\langle M^{2}\right\rangle^{2}}{L}
\end{aligned}
$$

where $M$ is the total magnetization in zero magnetic field of a strip of length $L$. These quantities should satisfy a finite size scaling form ${ }^{(17)}$

$$
\begin{aligned}
& m_{2} \sim n^{1+\gamma / v} f_{2}\left[n^{1 / v}\left(\beta-\beta^{c}\right)\right] \\
& m_{4} \sim n^{3+2 \gamma / v} f_{4}\left[n^{1 / v}\left(\beta-\beta^{c}\right)\right]
\end{aligned}
$$

which depends on the two exponents $\gamma$ and $v$.

In the limit $s \rightarrow 0$ this model gives the problem of SAW. ${ }^{(1,2)}$ In this limit the partition function of a system of $V$ spins can be rewritten as ${ }^{(1,2)}$

$$
Z(\beta, h)=\left(1+\frac{h^{2}}{2}\right)^{V}\left\{1+\sum_{\substack{p \geqslant 1 \\ l \geqslant p}} h^{2 p} \beta^{l}\left(1+\frac{h^{2}}{2}\right)^{-p-l} \Omega_{p l}\right\}
$$

where $\Omega_{p l}$ is the number of different configurations of $p$ nonintersecting SAWs of total length $l$ on the lattice of $V$ spins.

In this formula the factors $h^{2} / 2$ can be interpreted as the contribution of the SAWs of zero length. Such factors were omitted in early discussions. ${ }^{(2)}$ They do not affect the critical behavior of the infinite system but modify the finite size data. 
Using this geometrical interpretation, the partition function (13) can be calculated for strips of width $n$ and periodic boundary conditions by the transfer matrix technique. The calculation is similar to what was done in Ref. 8 , with the difference that there are now several polymers on the strip. In this case the possible configurations at one column $L$ are defined by the pairs of sites connected by the left part of the strip and by all the sites which are extremities of polymers whose other extremity is in the left part of the strip. The number of these configurations grows more rapidly than in Ref. 8. When calculating the transfer matrix elements which are defined in a similar way as in Ref. 8, one now has to take into account the polymers which begin or end when one goes from one column to the next. There is a factor $\beta$ for each monomer and $h$ for each end point of a polymer. These matrix elements depend thus on $\beta$ and $h$.

The partition function is simply obtained as a power of the largest eigenvalue of the transfer matrix $\lambda(\beta, h)$ and the moments $m_{2}$ and $m_{4}{ }^{(17)}$ are then given by

$$
\begin{aligned}
& m_{2}=\left.\frac{d^{2}}{d h^{2}} \log \lambda(\beta, h)\right|_{h=0} \\
& m_{4}=\left.\frac{d^{4}}{d h^{4}} \log \lambda(\beta, h)\right|_{h=0}
\end{aligned}
$$

These moments have a simple geometrical interpretation

$$
\begin{aligned}
& \frac{m_{2}(\beta)}{n}=\sum_{l=0}^{\infty} \omega_{l} \beta^{l} \\
& \frac{m_{4}(\beta)}{6 n}=-\sum_{l, l^{\prime}=0}^{\infty} \omega_{l, l^{\prime}} \beta^{l+l^{\prime}}
\end{aligned}
$$

In these formulas $\omega_{l}$ is the number of configurations of a SAW of length $l$ with a fixed origin (because we consider strips with periodic boundary conditions, this number does not depend on the position of this extremity on the strip). $\omega_{l, l^{\prime}}=\omega_{l^{\prime}, l}$ is the number of configuration of two SAWs of $l$ and $l^{\prime}$ links with at least one site in common, the extremity of one of the two walks being fixed. In evaluating these quantities one must give a special weight $\frac{1}{2}$ to the SAWs of zero length.

We note that one can obtain the $\omega_{l}$ or $\omega_{l, l^{\prime}}$ from the knowledge of $m_{2}$ and $m_{4}$. In particular, the asymptotic behavior of $\omega_{l}$ and $\omega_{l, l^{\prime}}$ for $l, l^{\prime}$ large is simply related to the singularities of $m_{2}$ and $m_{4}$.

It is difficult to accurately calculate derivatives when one performs these derivatives numerically. To avoid this difficulty we have performed all 
the calculations perturbatively as explained in Ref. 17, up to fourth order in $h$. This gives the derivatives of an eigenvalue with the same accuracy as the eigenvalue itself.

From knowledge of $m_{2}$ and $m_{4}$ one can get estimates of the critical properties using the finite size scaling form (12). If one defines

$$
R_{n}=\frac{m_{4}}{\left[m_{2}\right]^{2}}(\beta)
$$

One has, from (12)

$$
R_{n} \sim n f\left[n^{1 / \nu}\left(\beta-\beta^{c}\right)\right]
$$

An estimate $\beta_{n}^{c}$ of $\beta^{c}$ is then obtained by solving equations similar to (4)

$$
\frac{R_{n}\left(\beta_{n}^{c}\right)}{n}=\frac{R_{n-1}\left(\beta_{n}^{c}\right)}{n-1}
$$

Once $\beta_{n}^{c}$ is known one can get estimates of $v$ by the relation

$$
1+\frac{1}{v_{n}}=\frac{\log \left[\frac{d R_{n}}{d \beta}\left(\beta_{n}^{c}\right) / \frac{d R_{n-1}}{d \beta}\left(\beta_{n}^{c}\right)\right]}{\log [n / n-1]}
$$

and of the ratio $\gamma / v$ by

$$
1+\left(\frac{\gamma}{v}\right)_{n}=\log \left[\frac{m_{2, n}\left(\beta_{n}^{c}\right)}{m_{2, n-1}\left(\beta_{n}^{c}\right)}\right] / \log \left(\frac{n}{n-1}\right)
$$

Our results are given in Table IIA for strips oriented in the normal direction and IIB for strips in the diagonal direction.

One can see that the convergence of these results is rather slow, particularly for the exponent $v$ in the case of normal strips. The convergence is better for the ratio $\gamma / v$, although the use of an a posteriori or a priori analysis $^{(15)}$ does not improve the results significantly in this case. A plot of the quantities $\gamma / v$ versus $n^{-1,5}$ gave rather regular curves for the data, as shown in Fig. 2. From this figure we deduce

$$
\frac{\gamma}{v}=1.80 \pm 0.01
$$

The ratio $\gamma / v$ is thus obtained with a relative error of about $5.10^{-3}$ in this case. 
Table II. Results of a Finite Size Scaling Analysis of the Ratios $R_{n}(16)$ in the Case of Normal and Diagonal Strips ${ }^{a}$

\begin{tabular}{|c|c|c|c|c|c|}
\hline$n$ & $S_{n}$ & $\beta^{c}=\frac{1}{\mu_{\mathrm{SQ}}}$ & $\frac{\gamma}{v}$ & $v$ & $\frac{-m_{4}}{6 n m_{2}^{2}}$ \\
\hline \multicolumn{6}{|c|}{ A } \\
\hline 2 & 4 & 0.375807 & 2.14267 & 0.514706 & 1.37522 \\
\hline 3 & 6 & 0.371796 & 1.85941 & 0.632300 & 1.32887 \\
\hline 4 & 13 & 0.375621 & 1.81930 & 0.676755 & 1.41517 \\
\hline 5 & 23 & 0.377576 & 1.81261 & 0.701876 & 1.49017 \\
\hline 6 & 58 & 0.378389 & 1.81063 & 0.716286 & 1.53642 \\
\hline 7 & 130 & 0.378725 & 1.80873 & 0.724873 & 1.56227 \\
\hline 8 & 356 & 0.378875 & 1.80674 & 0.73035 & 1.57682 \\
\hline \multicolumn{2}{|c|}{ Expected values } & $\begin{array}{c}0.3790528 \\
\pm 0.0000025^{(5)}\end{array}$ & $\frac{43}{24}=1.79167^{(11)}$ & $\frac{3}{4}=0.75^{(11)}$ & \\
\hline \multicolumn{6}{|c|}{ B } \\
\hline 2 & 6 & 0.391467 & 2.01285 & 0.710675 & 2.27300 \\
\hline 3 & 12 & 0.381588 & 1.88918 & 0.714420 & 1.81893 \\
\hline 4 & 33 & 0.379971 & 1.84878 & 0.725042 & 1.71899 \\
\hline 5 & 79 & 0.379479 & 1.82978 & 0.731715 & 1.67710 \\
\hline 6 & 249 & 0.379117 & 1.82035 & 0.73642 & 1.65280 \\
\hline \multicolumn{2}{|c|}{ Expected values } & $\begin{array}{c}0.3790528 \\
\pm 0.0000025^{(5)}\end{array}$ & $\frac{43}{24}=1.79167^{(11)}$ & $\frac{3}{4}=0.75^{(11)}$ & \\
\hline
\end{tabular}

${ }^{a} S_{n}$ is the size of the symmetrized transfer matrix and $\mu_{\mathrm{SQ}}$ the connectivity constant (2) of the square lattice. The convergence, which is rather slow for the exponent $v$, is better for $\gamma / v$.

The quantity

$$
\lim _{n \rightarrow \infty} \frac{-m_{4}}{6 n m_{2}^{2}}\left(\beta^{c}\right)
$$

is expected to be universal, depending only on the universality class of the model and on the finite size scaling geometry (strips with periodic boundary conditions in this case).

Such ratios have already been calculated by various methods in the case of the 2D and 3D Ising Model $^{(16-18)}$ or 2D and 3D percolation. ${ }^{(17)}$ Mean field values and $\varepsilon$ expansion results have also been given. ${ }^{(19)}$ From the data given in Table II, we get the following estimate for this universal ratio in the case of polymers

$$
\mathscr{R}=\lim _{n \rightarrow \infty} \frac{-m_{4}}{6 n m_{2}^{2}}\left(\beta^{c}\right)=1.63 \pm 0.03
$$




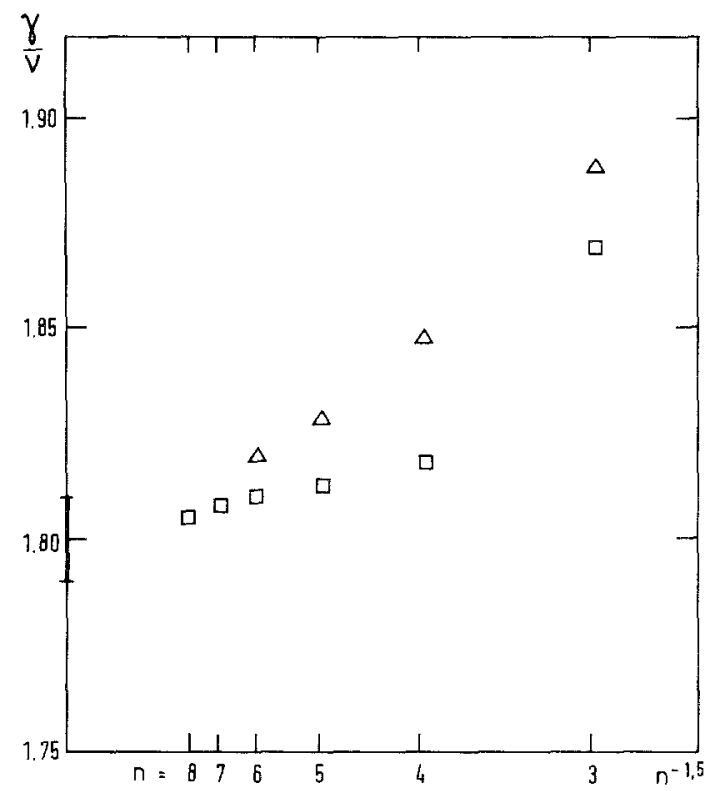

Fig. 2. Plot of our estimates of $y / v$ (see Table II) versus $n^{-1,5}: \square$ - strips in normal direction; $\triangle$-strips in diagonal direction. The result of the final extrapolation is indicated with error bars.

This ratio has a simple physical meaning. One knows ${ }^{(1,2)}$ that the partition function (13) is the grand canonical partition function of a polymer solution where the lengths of the chains and their concentrations fluctuate. For a polymer density $c$ (we take the lattice spacing as unit length) and a temperature $T$, the virial expansion of the osmotic pressure reads

$$
\frac{\pi}{T}=c+b c^{2}+\cdots
$$

In this expansion $T$ is the temperature of the polymer solution. It is not related to the properties of the magnetic system whose temperature $\tau=1 / \beta$ controls the mean length $\mathscr{L}$ of the chains. The coefficient $b$ for a solution of polymers on strips is then given by ${ }^{(1,2,20)}$

$$
b_{n}=n^{2}\left(\frac{-R_{n}(\beta)}{6 n}\right)
$$

When $\beta$ goes to $\beta^{c}$, the coefficient $b$ for an infinite system diverges with $\mathscr{L}$ like

$$
b \sim \mathscr{L}^{2 v}
$$


On the strip, it has thus the scaling behavior

$$
\lim _{n \rightarrow \infty} \frac{b_{n}\left(\beta^{c}\right)}{n^{2}}=\mathscr{R}
$$

If one does not want to work with polymers of zero length, ${ }^{(1,2)}$ the virial coefficient on strips is no more given by $b_{n}$. However when $\beta$ goes to $\beta^{c}$, this coefficient depends on very long polymers only, so the formula (26) is still valid. The universal ratio $\mathscr{R}$ is thus simply related to the scaling behavior of the second virial coefficient in the case of polymers.

In conclusion we have developed two independent transfer matrix methods for the calculation of the exponent $\gamma$ in the SAW problem. Our first method is a simple application of the relation between the amplitude of the correlation length at the critical point and the exponent $\eta$, while our second method involves the calculation of properties of strips with several polymers. The results (9) and (21) of these two methods are both compatible with the conjectured value ${ }^{(11)}$ of the ratio $\gamma / v=43 / 24=1.79167$, and they both exclude the old value ${ }^{(3)} \gamma / v=16 / 9=1.77777$. Our best result (9) compares well with the precision of the most recent series analysis. ${ }^{(5)}$ The result (21), although less satisfactory, is comparable in precision to the estimates of less extensive series analysis ${ }^{(4)}$ or of Monte Carlo simulations. These two methods thus provide a good way of determining the exponent $\gamma$ by the transfer matrix method in the case of SAWs. They could easily be extended to other situations like the $\Theta$ point of $2 \mathrm{D}$ linear polymers. We hope to do such a calculation in the next future. The first method using conformal invariance could also be extended to the determination of other exponents by looking at the other eigenvalues of the transfer matrix. The second method using several polymers could be used also in the study of the properties of polymer solutions.

\section{REFERENCES}

1. P. G. De Gennes, Phys. Lett. 38A:339 (1972); J. Des Cloizeaux, J. Phys. 36:281 (1975).

2. G. Sarma, in Les Houches 78, Ill Condensed Matter (R. Balian et al., eds.), (North Holland, Amsterdam, 1978); P. D. Gujrati, Phys. Rev. A24:2096 (1981); J. C. Wheeler and P. Pfeuty, Phys. Rev. A24:1050 (1981).

3. I. Majid, Z. V. Djordjevic, and H. E. Stanley, Phys. Rev. Lett. 51:143 (1983).

4. A. J. Guttman, J. Phys. A17:455 (1984).

5. I. G. Enting and A. J. Guttman, J. Phys. A18:1007 (1985).

6. D. C. Rapaport, J. Phys. A18:L39 (1985); A18:L201 (1985).

7. A. Beretti and A. D. Sokal, J. Stat. Phys. 40:483 (1985).

8. B. Derrida, J. Phys. A14:L5 (1981).

9. S. Redner and P. J. Reynolds, J. Phys. A14:2679 (1981). 
10. K. Kremer and M. N. Barber, J. Phys. A17:L215 (1984).

11. B. Nienhuis, Phys. Rev. Lett. 49:1062 (1982).

12. P. Flory, Principles of Polymer Chemistry (Cornell University Press, Ithaca, New York, 1967).

13. M. N. Barber, in Phase Transitions and Critical Phenomena, Domb and Lebowitz, eds., vol. 8 (Academic Press, London, 1983).

14. J. L. Cardy, J. Phys. A17:L385 (1984).

15. B. Derrida and D. Stauffer, J. Phys. 46:1623 (1985).

16. A. D. Bruce, J. Phys. C14:3667 (1981); K. Binder, Z. Phys. B43:119 (1981); M. N. Barber, R. B. Pearson, D. Toussaint, and J. L. Richardson, Phys. Rev. B32:1720 (1985).

17. H. Saleur and B. Derrida, J. Phys. 46:1043 (1985).

18. T. W. Burkhardt and B. Derrida, Phys. Rev. B32:7273 (1985).

19. E. Brézin and J. Zinn-Justin, Nucl Phys. B257:(FS14), 867 (1985).

20. D. J. Burch and M. A. Moore, J. Phys. A9:451 (1976). 